\title{
Le commerce de l'eau virtuelle : du concept à la politique
}

Trade in virtual water: from the concept to a policy

Lysiane Roch et Corinne Gendron

\section{(2) OpenEdition}

\section{Journals}

Édition électronique

URL : http://journals.openedition.org/geocarrefour/1259

DOI : 10.4000/geocarrefour.1259

ISSN : 1960-601X

Éditeur

Association des amis de la Revue de géographie de Lyon

Édition imprimée

Date de publication : 1 octobre 2005

Pagination : 273-284

ISSN : $1627-4873$

Ce document vous est offert par Université du Québec à Montréal

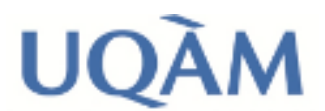

Université du Québec à Montréal

Référence électronique

Lysiane Roch et Corinne Gendron, "Le commerce de l'eau virtuelle : du concept à la politique ", Géocarrefour [En ligne], vol. 80/4 | 2005, mis en ligne le 01 juin 2009, consulté le 06 juin 2019. URL: http://journals.openedition.org/geocarrefour/1259; DOI : 10.4000/geocarrefour.1259 


\section{Lysiane ROCH}

École des sciences de la gestion Université du Québec à

Montréal

\section{Corinne GENDRON}

École des sciences de la gestion Université du Québec à Montréal

\section{RÉSUMÉ}

Alors qu'un nombre

grandissant de pays fait face

à un problème de pénurie

d'eau, de nouveaux moyen

d'augmenter l'offre ou de

réduire la demande tendent à émerger. C'est ainsi qu'on a pu remarquer que dans certaines régions, les importations alimentaires augmentent au fur et à mesure que les ressources mesure que les ressources en eau s'epuisent. De ce constat est né le concept d'eau virtuelle, qui cherche à
rendre compte du rôle que rendre compte du international dans un contexte de pénurie d'eau. L'eau virtuelle peut se définir comme l'eau qu'a nécessitée la production d'un bien. Lorsqu'un pays importe ce bien, des denrées agricoles par exemple, il n'a pas à utiliser l'eau qui aurait été utiser Yeau qui aurait éte necessaire à sa production. Sachant que les ressources en eau sont très inégalement réparties sur la planète et que l'agriculture est le plus grand consommateur mondial d'eau, peut-on considérer le commerce de l'eau virtuelle comme un moyen potentiellement efficace de diminuer les pénuries et leurs conséquences? Nours consequences ? Notre analyse quien seulement le commerce de l'eau virtuelle constitue une stratégie présentement pe appliquée, mais que son potentiel face aux défis de la rareté de l'eau est très faible lorsqu'on considère le contexte politico-économique dans lequel il prend place.

\section{MOTS-CLÉS}

Eau virtuelle, pénurie d'eau, gestion de l'eau, commerce international, agriculture

\section{ABSTRACT}

As a growing number of

\section{Loommoreo do Fead vturlo: du eoneept a la polípro}

À l'heure actuelle, environ 80 pays font face à des pénuries d'eau chroniques (Lasserre, 2002) ${ }^{1}$. Selon la Population Action International, 436 millions de personnes vivaient dans une situation de stress hydrique et de pénurie d'eau en 1997, et on prévoit qu'ils seront cinq fois plus nombreux en 2050 Quant au directeur de I'International Food Policy Research Institute, il estime qu'un pays sur cinq fera face à une grave pénurie d'ici 25 ans ( $D e$ Villiers, 2000). De nombreux facteurs permettent en effet de croire que le problème de la pénurie ira en s'aggravant: croissance démographique aumentation du niveau de vie te augmentation du niveau de vie et accroissement de l'irrigation pour cultiver des terres en perte de productivité n'en sont que quelques exemples. Comment les pays en situation de stress hydrique ou de pénurie gèrent-ils cette situation? Au Moyen-Orient, première région à avoir manque d'eau, les réserves ne suffisent plus pour répondre aux besoins industriels, agricoles et domestiques depuis 1970 (Allan, 1997). Certains pays de la région sont en situation de déficit depuis plus longtemps encore. La péninsule arabique, Israël et la Palestine ont franchi ce seuil critique au cours des années 1950, la Jordanie dans les années 1960 et l'Égypte dans les années 1970. Pourtant, les politiciens de la region nient aujourd'hui etre politiciens dé la region nient aujourd'hui être en

Cette situation en apparence paradoxale s'explique lorsqu'on observe l'évolution des importations de céréales au Moyen-Orient. Alors qu'environ 10 millions de tonnes de céréales étaient importées dans la région au début des années 1970, ce chiffre s'élève à plus de 40 millions de tonnes au milieu des années 1980 (Allan, 2001). En considérant qu'en moyenne l'agriculture est responsable de 70 à $75 \%$ des prélèvements d'eau à l'échelle mondiale 2002a) on comprend facilement (Losserre, rooral, on comprend facilement comment laugrations agricoles peut reduire drastiquement les besoins en eau d'un pays et mesme donner l'impression qu'il possèd de l'eau en quantité suffisante.

Cet exemple illustre bien le rapport étroit qui lie pénurie d'eau et activités commerciales. Le échanges de produits dont la production demande beaucoup d'eau peuvent indirectement accentue ou diminuer la pression sur la ressource d'une région, non pas en augmentant l'offre, comme c'est le cas avec les dérivations et importations ${ }^{2}$, mais plutôt en réduisant la demande dans un mais plutot en réduisant la demande dans un secteur donn. Dans les annees 1990, professeur John Ans les annees 1990, le concept d'eau phénomène. L'eau virtuelle peut se définir comme l'eau qu'a nécessitée la production d'un bien. Produire une tonne de céréales, par exemple, demande $1000 \mathrm{~m}^{3} \mathrm{~d}^{\prime}$ eau. Pour un pays en situation de pénurie, il est plus facile de se procurer une tonne de céréales que les $1000 \mathrm{~m}^{3}$ d'eau qui ont été nécessaires à leur production. travers le commerce international, cette eau virtuelle se voit transférée des pays riches en eau vers les pays en situation de pénurie (Allan, 1996). On parle alors de commerce d'eau virtuelle.

Le concept introduit par Allan est d'abor explicatif : il permet de rendre compte d'u phénomène, d'une dimension de la réalité jusqu'alors trop souvent omise dans les analyses. Par exemple, plus d'eau coule des États-Unis et de la Communauté Européenne vers le Moyen-Orient et l'Afrique du Nord sous forme d'eau virtuelle qu'il n'en coule dans le Nil pour l'agriculture egyptienne chaque année (Allan, 1996). De ce fait, alors que l'urgence du problème de pénuries d'au et de ses lourdes consequences humaines et écosystemiques se fait sentir, le commerce de l'eau virtuelle est de plus en plus presenté comm une stratégie permettant de pallier ces pénuries.

EAU VIRTUELLE : DU CONCEPT EXPLICATIF À LA STRATEGIE POLITIOUE

Le concept d'eau virtuelle met en évidence l'eau qui entre dans le processus de production des biens qu'on consomme. Nombre d'auteurs se sont intéressé à quantifier l'eau virtuelle de différents produits, c'est-à-dire l'eau qui a été nécessaire pour les produire. Ces calculs ont ensuite pu mener à la quantification de l'eau indirectement échangée par le biais du commerce, ou commerce de l'eau virtuelle. Si, jusque là, le concept avait un vocation principalement explicative, nous verrons $q^{\prime} u^{\prime} i l$ tend à prendre une dimension de plus en plus politique.

Quantification de l'eau virtuelle d'un produit

L'intérêt du concept d'eau virtuelle vient d'abord de sa capacité à reconnaître l'eau comme facteur de production. En quantifiant l'eau nécessaire à production d'un bien, il devient possible d'établi le rapport entre l'usage de l'eau comme facteur de production et la consommation d'un individu ou d'une nation. Alors qu'un être humain a besoin de boire de 2 à 5 litres d'eau par jour et nécessite de 25 à 100 litres pour ses usages domestiques, il a besoin de 1000 à 6000 litres par jour pour se nourrir La fraction invisible de l'eau soit celle soit celle qu'on retrouve dans les aliments, est donc de 37 57 for plus grande que la fraction visible (Turton 2000). Or, la fraction invisible est largement ignorée dans la littérature (Turton, 2000). Le concept d'eau virtuelle permet de la mettre en évidence.

Plusieurs chercheurs se sont intéressé à quantifier l'eau nécessaire à la production de certains biens. En raison de la large part qu'occupe l'agriculture dans la consommation d'eau, ce son principalement les denrées agricoles qui ont fait l'objet de ces calculs. Le calcul de l'eau virtuelle d'un produit soulève d'abord des questions 
méthodologiques. La définition même qu'on donne à l'eau virtuelle aura une incidence sur son calcul. Dans une première approche, l'eau virtuelle est définie comme l'eau qui a été réellement utilisée pour produire un bien (Hoekstra, 2003) Dans une deuxième approche, l'eau virtuelle se définit comme l'eau qui aurait été nécessaire pour produire un bien dans les conditions du pays où î est consommé. Ces deux définitions presentent une différence importante, puisque la production d'une même quantité de céréales peut exiger de deux à trois fois plus d'eau dans un pays aride que dans un pays au climat humide (Hoekstra, 2003). dans un pays au clima huld (2003). On peut rays au clima humide (Hoekstra, 2003). approche lorsqu'on tente de calculer l'eau virtuelle d'un bien qui ne peut pas être produit par le pays importateur (Hoekstra, 2003). On ne peut, pa exemple, calculer l'eau qui aurait été nécessair pour faire pousser du riz en Allemagne, puisqu'on ne peut pas cultiver de riz dans ce pays. Face cette problématique, Renault (2003) propose le principe d'équivalence nutritionnelle, qui consiste à calculer l'eau nécessaire à la production d'une denrée procurant des éléments nutritifs équivalents.

Les cultures pluviales ne consomment que de l'eau verte ${ }^{3}$, tandis que les cultures irriguées consomment à la fois de l'eau verte et de l'eau bleue $^{4}$ (Renault, 2003). Pour quantifier I'eau consommée par une plante pendant sa croissance, consommée par une plante pendant sa croissance,
Renault (2003) propose d'évaluer cette Renault (2003) propose d'évaluer cette consommation à partir de l'évapotranspiration de I'eau (qui inclut l'eau verte et l'eau bleue). Oki et Kanae (2004), quant à eux, ont choisi d'inclure l'eau qui s'évapore des cultures, du champ luimême ainsi que de l'eau qui s'infiltre dans le sol, si cette dernière est nécessaire à la culture.

Différents facteurs doivent être pris en considération dans le calcul de l'eau virtuelle d'un produit. Ainsi, on doit considérer le lieu et la période de production, l'endroit où la mesure est effectuée, le mode de production et son efficacité ${ }^{5}$ ainsi que la prise en compte de l'eau utilisée dan l'ensemble du processus de production pour

lequel on effectue le calcul6 (Hoekstra, 2003). Les résultats des différents chercheurs varient selon ces facteurs et la définition qu'ils donnent de l'eau virtuelle. Hoekstra (2003) a compilé les résultats des calculs de l'eau virtuelle contenue dans certains produits (tabl. 1).

Pour faire face aux fluctuations des conditions climatiques, les aliments peuvent être stockés pendant les bonnes saisons et utilisés ensuite lorsque les conditions sont moins bonnes. De l'eau ayant été nécessire à la production ces aliments, on peut considerer que leur entreposage constitue une 203). A l'écheller mond virtuelle (Renault, 2003). A l'échelle mondiale, ces reservoirs représenteraient 4000 milliards de $\mathrm{m}^{3}$ d'eau ${ }^{10}$ (Renault, 2003).

La quantification de l'eau virtuelle d'un produit mation nécessite une quantité d'eau spécifique. La consommation de bœuf exige environ dix fois plus d'eau que la consommation d'un poids équivalent de blé. II est évident qu'à poids égal deux alme apport nutrion d'équivalence nutritionnelle est fort utile. Selon ce principe, avec $1 \mathrm{~m}^{3}$ d'eau utilse pour produire des pommes de terre, on obtient un apport énergétique équivalent à une quantité de blé dont la culture aurait nécessité $2,5 \mathrm{~m}^{3} \mathrm{~d}$ 'eau (Renault, 2003).

Avec le concept d'eau virtuelle et le principe d'équivalence nutritionnelle, on peut calculer l'eau nécessaire pour soutenir différents régimes. Ainsi, on constate que le régime alimentaire moyen aux États-Unis correspond à une consommation de $5,4 \mathrm{~m}^{3}$ d'eau par personne et par jour (Renault et Wallend 2000). En comparaison, un régime Wécéterien 11 . égersonne et par jour et un regime minimal pour repondre aux besoins biologiques de base $1 \mathrm{~m}^{3}$ (Renault et Wallender, 2000)

Pour rendre compte de la quantité d'eau nécessaire pour soutenir un mode de consom-

Tableau 1 : Contenu en eau virtuelle de certains produits, en $\mathrm{m}^{3} /$ tonne

(Traduction libre de Hoekstra, 2003, p.16)
\begin{tabular}{|l|r|r|r|r|}
\hline & $\begin{array}{r}\text { Hoekstra } \\
\text { \& Hung (2003) }\end{array}$ & $\begin{array}{r}\text { Chapagain } \\
\text { \& Hoekstra (2003) }\end{array}$ & $\begin{array}{r}\text { Zimmer } \\
\text { \& Renault (2003) }\end{array}$ & Oki et al. (2003) \\
\hline Blé & 1150 & - & 1160 & 2000 \\
Riz & 2656 & - & 1400 & 3600 \\
Maïs & 450 & - & 710 & 1900 \\
Pommes de terre & 160 & - & 105 & - \\
Soja & 2300 & - & Égypte $: 2750$ & 2500 \\
Boeuf & - & 15977 & 13500 & 20700 \\
Porc & - & 5906 & 4600 & 5900 \\
Volaille & - & 2828 & 4100 & 4500 \\
Oeufs & - & 4657 & 2700 & 3200 \\
Lait & - & 865 & 790 & 560 \\
Fromage & - & 5288 & - & - \\
\hline
\end{tabular}

countries are facing water scarcity, new strategies which try to increase supply or to reduce demand are emerging. Thus, it is noted that in some regions, food imports are increasing as water reserves decrease. From this observation the concept of virtual water was introduced, which tries to demonstrate the role played by international trade in a water scarcity context. Virter scarcity context. as the water can be defined as the water necessary to produce a good. When a agricultural produce for example, it does not have to use the water needed for its production. Knowing that water resources are unevenly distributed and that agriculture is the world's greatest water user, can we consider virtual water trade as a potentially efficient means to reduce scarcity and its scarcity and its
consequences? Our analysis shows rather that not only is virtual water trade a seldom applied strategy, but that its potential to provide a better response to basic human and ecosystem needs is very low when considering the political and economic contexts in which it takes place.

\section{KEY WORDS}

Virtual water, water scarcity water management international trade, agriculture. 
1 - Les auteures tiennent à remercier les relecteurs pour leur contribution à l'enrichissement de cet article.

2 - Le coût particulièrement élevé des dérivations massives ainsi que leurs conséque nces écologiques constituent des obstacles majeurs à leur réalisation. Quant au transport de l'eau par navires-citernes et grands sacs de plastique, leur coût et les obstacles techniques n'en font qu'une soluniques n'en font qu'une solution L'appoint (Lasserre, 2002a). Lidee du c virtuelle peut être vue comme une alternative plus réaliste au rêve de commerce international d'eau réelle. Des ingénieurs comme Alan Conley, par exemple, privilégient l'idée d'échanger des denrées alimentaires plutôt que de recourir à de nouveaux projets hydrauliques tels les transferts inter-bassins (Allan, 2003).

3 - L'humidité du sol est considérée comme de l'eau verte (Falkenmark, 1997).

4 - On qualifie "d'eau bleue" I'eau des aquifères et des cours d'eau (Falkenmark, 1997)

5 - Cela implique de considérer s'il y a un gaspillage d'eau dans le processus duction.

6 - Ainsi, I'eau virtuelle contenue dans un animal vivant tenue dans un animal vivant I'eav qu'il a bue, leau qu'll a bue, mais aussi de la nourriture qu'il a consommé durant sa vie, et donc de l'eau qui a été nécessaire pour produire le grain. De plus, une même vache pourra à la fois donner du lait de la viande et du cuir. Dans ce cas, l'eau du cuir. Dans ce cas, l'eau I'anima vivant doit etre divise Vanima vivant doit etre divisee qu'on en retire (Hoekstra, qu'on

7 - Ces chiffres représentent une moyenne globale (Hoekstra, 2003). mation donné, on a proposé le concept d'empreinte en eau. Développé par analogie avec le concept d'empreinte écologique, l'empren avec le concept d'empreinte écologique, l'empreinte en eau d'un individu, d'une entreprise ou d'une nation peut se définir comme le volume total d'eau douce nécessaire pour produire les bien consommés par cet individu, cette entreprise ou cette nation (Chapagain et Hoekstra, 2004). II s'agit alors non seulement de calculer la consommation d'eau de surface, d'eau souterraine et d'eau contenue dans le sol, mais aussi de considérer l'eau nécessaire pour produire les biens consommés ou importés par la nation, l'individu ou l'entreprise. Dans leur étude, Chapagain et Hoekstra (2004) font ressortir les contrastes entre los empreintes en rau des différents pays 12 . 'emp pays ${ }^{12}$. lemp est de $2480 \mathrm{~m}^{3}$ d'eau par

La quantification de l'eau virtuelle de différents produits permet donc d'établir un rapport plus direct entre la consommation de certains biens et l'eau qui est intervenue dans leur production. Le commerce de l'eau virtuelle viendra quant à lui transposer ce rapport à l'échelle de la consommation des pays. Les biens consommés à l'intérieur d'un pays impliquent que de l'eau a été nécessaire pour les produire: : atte eau peut venir du pays luipour les produire : cette eau peut venir du pays luimême, mais aussi d'un autre pays, si le produit a
été importé.

\section{De l'eau virtuelle au commerce de l'eau virtuelle}

À travers les échanges commerciaux, des biens dont la production a demandé une certain quantité d'eau sont importés et exportés entre différents pays, donnant lieu à des échanges d'eau virtuelle, ou commerce de l'eau virtuelle 13 Plusieurs groupes de recherche se sont intéressé à quantifier ces échanges. Hoekstra et Hung de quantifier ces échanges. Hoekstra et Hung, de I'UNESCO-IHE, ont estimé que la circulation internationale d'eau virtuelle associée à des récoltes était de $695 \mathrm{~km}^{3}$ par annee (Hoekstra et Hung, 2005) et celle associée à des produits
animaux était de $245 \mathrm{~km}^{3}$ par année ${ }^{14}$, pour un animaux était de $245 \mathrm{~km}^{3}$ par année ${ }^{14}$, pour un Hoekstra, 2003). Un groupe de recherche japonais a effectué le calcul et arrive à deux résultats différents selon qu'ils se mettent dans la perspective du pays exportateur $\left(683 \mathrm{~km}^{3}\right.$ par année $)^{15}$ ou du pays importateur (1 $138 \mathrm{~km}^{3} \mathrm{par}$ année) ${ }^{16}$ (Oki et Kanae, 2004). Zimmer et Renault dans une étude de la FAO, ont estimé de leur côté que la circulation d'eau virtuelle à l'échelle internationale serait de $1340 \mathrm{~km}^{3}$ par année ${ }^{17}$ pour I'an 2000 (Zimmer et Renault, 2003). Les échanges sous forme d'eau virtuelle représenteraient alors $26 \%$ de l'eau virtuelle totale à l'échelle mondiale (Zimmer et Renault, 2003)

II faut noter que ces chercheurs ont pris en compte l'eau virtuelle des produits agricoles, de même que des produits alimentaires, à l'exception du tabac du coton (Hoekstra et Hung, 2005) et du cuir (Chapagain et Hoekstra, 2003). D'autres cultures consomatrictes fleurs en le bois. II faut d'aileus rapeler ques feurs et le bois. Il faut dailleurs rappeler que la production de certains produits non agricoles, tels les puces electroniques et les voitures, demand aussi beaucoup d'eau. Un calcul de la consom mation d'eau pour l'ensemble des produits agricoles ou manufacturés augmenterait sans doute les quantités d'eau virtuelle échangées par le commerce.

À la lumière de ces études, il est possible de degager les grands flux d'eau virtuelle entre les différentes régions du monde. Ainsi, les principaux pays exportateurs sont les États-Unis, le Canada, la Thaïlande, I'Argentine et I'Inde, tandis que les principaux pays importateurs sont le Si Lanka principaux pays importateurs sont le Sri Lanka, Japon, et les Pays-Bas (Hoekstra et Hung, 2002) L'Amérique du Nord est de loin la région du monde qui exporte le plus d'eau virtuelle, tandis que l'Asie Centrale et l'Asie du Sud en sont les plus grands importateurs (Hoekstra et Hung, 2005). De façon générale, les pays en développement sont moins stables, la quantité d'eau virtuelle importée ou exportée pouvant varier de façon importante d'une année à l'autre (Hoekstra et Hung, 2005)

Avec le concept d'eau virtuelle, il est donc possible d'expliquer comment le système économique d'expliquer commet à des pays de réagi à une international perme à des pays de reagir à un situation de rarete de l'eau en important de produits agricoles. II met en évidence le rôle peu visible du commerce international dans les politiques de gestion de l'eau, particulièrement au Moyen-Orient et en Afrique du Nord. Ainsi, Israë importe 13 fois plus de céréales qu'elle n'en produit, la Lybie 10 fois plus, I'Algérie 2 fois plus et I'Égypte et le Maroc en importent 0,6 fois plus (Yang et Zehnder, 2002)

Dimension normative du concept d'eau virtuelle

Les concepts d'eau virtuelle et de commerce de l'eau virtuelle ont donc été introduits pour mettre en lumiere une dimension de la réalité qui échappait à plusieurs analyses. Allan (2003) considère qu'il peut servir à de nombreuses disciconsidere quill peut senir à de nombreuses disciplines : selon lui, des chercheurs de discipline aussi : aries que leconomie, I'ingenierie, le droit tionales pourraient aboutir à de fausses analyses, faute de prendre en considération le rôle de l'eau virtuell dans l'économie internationale. Le spectre des guerres de l'eau qui ne se sont pas réalisées en est un bon exemple ${ }^{18}$

Jusqu'alors principalement explicatif, on a pu observer récemment que le concept d'eau virtuell s'est vu approprié part des acteurs qui lui on conféré un nouveau sens et une nouvelle portée le faisant passer d'un statut "explicatif" à un statu 


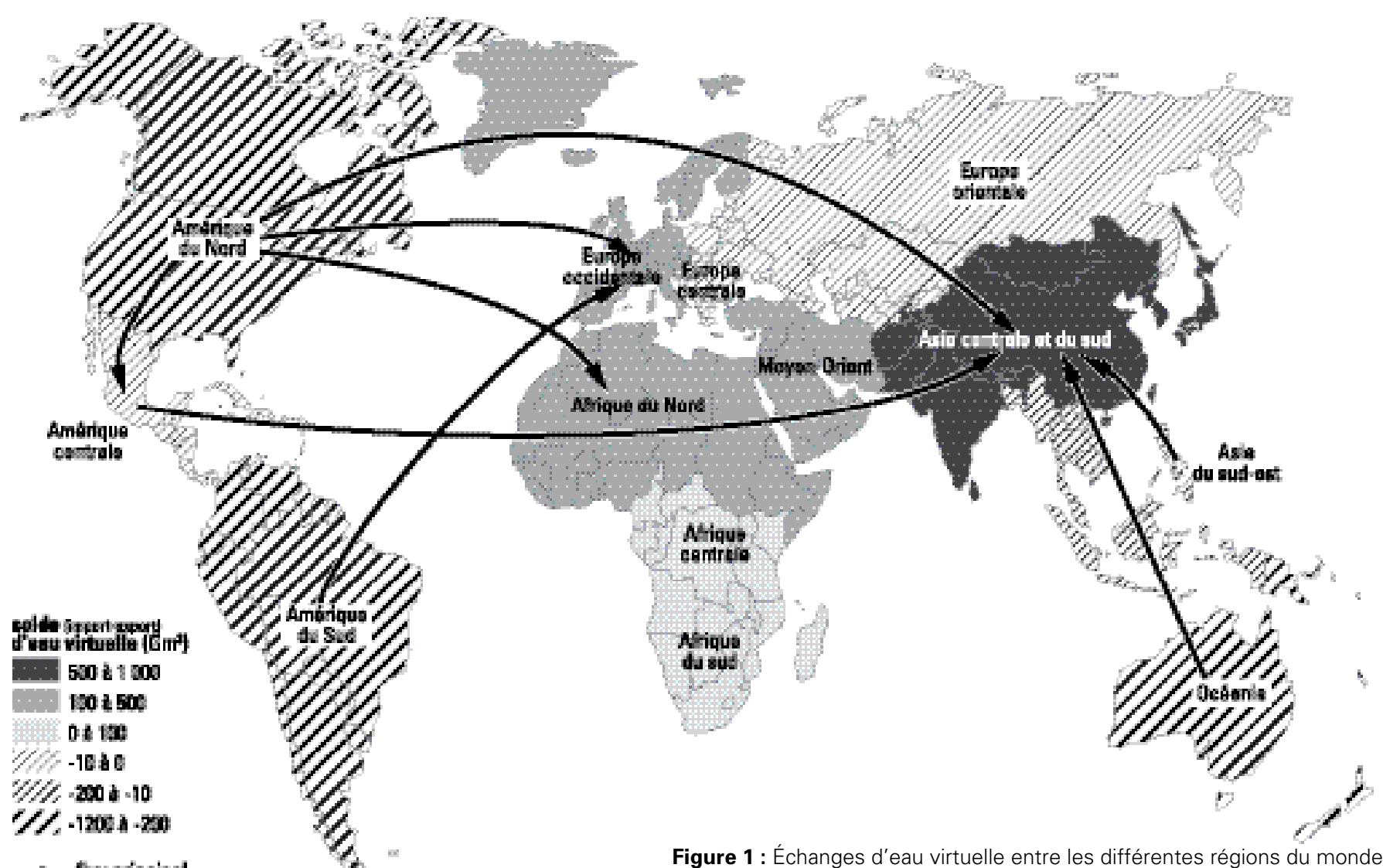

Figure 1 : Échanges d'eau virtuelle entre les différentes régions du monde Source : Hoekstra, 2003, p.18

"normatif"19. Le concept d'eau virtuelle est alors conçu comme une politique de gestion de l'eau qu'on souhaite appliquer comme solution à un certain nombre de problématiques. C'est ainsi

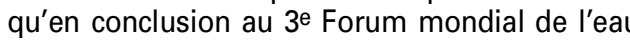
plusieurs organisations internationales et groupes de recherche ${ }^{20}$ se sont engagés non seulement à "poursuivre leurs efforts [...] afin de permettre un meilleure compréhension du concept d'eau virtuelle", mais aussi à fournir aux gouvernements les informations et les outils nécessaires a 'utilisation a commerce de l'eau virtuelle comme moven efficace de promouvoir sauvegarde de l'eau et de l'intégrer à part entière aux politiques nationales et régionales de protection de l'environnement, de gestion de l'eau et de l'alimentation (Secrétariat du troisièm forum mondial de l'eau et Conseil mondial de I'eau, 2005)

Même chez les chercheurs qui s'intéressent au concept, la frontière est souvent mince entre explication et prescription. II n'est pas anodin de rappeler que dès ses origines, on a utilis I'expression eau virtuelle "pour attirer l'attentio sur le fait que les sérieuses pénuries d'eau locales pourraient être améliorées efficacement par le processus économique global" (Allan, 2003, p. 4) Même si le phénomène n'est pas toujours flagrant on voit se dessiner une certaine propension normative dans l'argumentaire de nombre de chercheurs.
On attribue ainsi au commerce de l'eau virtuelle le potentiel de répondre aux pénuries locales (Yang et Zehnder, 2001) et de pallier la distribution inégale de l'eau entre les différents pays (Hoekstra et Hung, 2005; Turton, 2000). On estime qu'il pourrait ainsi prévenir les conflits (Bouwer, 2000) et les guerres de l'eau (Allan, 1996a) et qu'il permettrait d'assurer la sécurité alin (Shuval, 1999). On le prop moyen de réaliser des économies d'eau à l'échelle globale en produisant la nourriture là où chaque goutte d'eau est la plus efficace (Hoekstra et Hung, 2005)21. Enfin li pous ence 2005)21. Enfin, il constituerait une solution plus flexible que toutes les mesures d'approvisionnement conçues par les ingénieurs pour faire face à des fluctuations de l'offre suite, par exemple, à une mousson où les précipitations ont été moins abondantes que la moyenne (Allan, 2003).

Sur le plan économique, on considère que le commerce de l'eau virtuelle constitue un moyen d'approvisionnement en eau moins dispendieux que l'extraction de l'eau à partir d'aquifères profonds ou de systèmes d'irrigation profonds ou de systemes deirrigation moins permettrait aussi de maximiser la valeur de l'eau en la destinant à des usages plus rentables (Shuval, 1999). Ces usages peuvent être industriels, commerciaux ou touristiques (Shuval, 1999), mais peuvent aussi être agricoles, I'eau étant dans ce cas dirigée vers des cultures d'exportation plus
8 - A part pour le cas de I'Égypte, les données font référence à une étude pour Californie (Hoekstra, 2003).

9 - Les données font référence au Japon (Hoekstra, 2003).

10 - Ce chiffre comprend auss le sucre, l'huile, la viande et les animaux d'élevage vivants.

11 - Le régime végétarien a été établi en conservant le même apport en eufs et en beurre que dans le régime de base, alors que le lait représente $70 \%$ de la quantité du régime de base. L'équilibre est obtenu par l'augmentation de la quantité de produits végétaux à haute teneur nutritive.

12 - Quatre principaux facteurs expliquent les variations entre les empreintes en eau des différents pays : le volume de consommation moyen par personne (généralement relié au revenu national brut du pays), les habitudes de con- 
sommation de ses habitants, le climat et les pratiques agricoles. Ainsi, l'empreinte en eau élevée des États-Unis s'explique surtout par l'important niveau de consommation de viande et de produits industriels de ses habitants. L'Iran, de son côté, présente aussi une empreinte en eau relativement élevée, mais celle-ci s'explique plutôt par une faible productivité agricole et une évapotranspiration importante (Cha

13 - Si un pays importe des denrées provenant d'une culture pluviale plutôt que de les produire lui-même par une culture irriguée, on pourrait alors parler d'un transfert de consommation d'eau bleue vers une consommation d'eau verte.

14 - II s'agit aussi de moyennes pour la période de 1995 à 1999. L'étude est basée sur des données qui correspondent à l'eau virtuelle du pays exportateur.

15 - La différence avec les résultats de Chapagain et Hoekstra peut s'expliquer par le fait qu'Oki et Kanae ont intégré moins de produits à leur analyse (Hoekstra, 2003).

16 - Notons qu'Oki et Kanae utilisent I'expression "eau réelle", plutôt qu'"eau virtuelle", lorsqu'lis parlent de l'eau utilisée par les pays exportateurs, puisque cette eau a été réellement utilisée.

17 - Les résultats de Zimmer et Renault sont basés sur des données de 1990, qui ont été ajustées en supposant une augmentation de la productivité de l'eau de $1 \%$ par anné. L'é de l'eau de $1 \%$ par année. L'étude est baseesur des ent à l'eau virtuelle du pays importateur.

18 - L'ancien secrétaire général des Nations Unies, Boutros Boutros-Ghali et le roi Hussein rentables, telles que le coton, les fruits et les légumes ${ }^{22}$ (Oadir et al., 2003).

Si les arguments utilisés par ces chercheurs diffèrent, une tendance néanmoins se dessine dans leurs écrits, soit celle d'introduire le commerce de l'eau virtuelle comme solution à explorer pour faire face à la crise de l'eau qui menace de plus en plus de pays.

À I'heure où le concept d'eau virtuelle tend ainsi à glisser de la description à la prescription, il est fondamental de se questionner sur le potentiel ré du commere de potentiel ree du commerce de l'eau virtuelle en regard de la menace croissante des pénuries. Cela demande d'abord de mesurer à quel point le commerce de l'eau virtuelle est une stratégie réellement utilisée pour contrer les pénuries ou les déficits en eau. Ensuite, on peut se demander s'il s'agit d'une stratégie utilisable. Est-elle accessible à tous les pays ? Permettrait-elle réellement de redistribuer l'eau d'une façon plus équitable entre les pays et à l'intérieur de ces derniers, en répondant aux besoins de base des population et des écosystèmes ? Se pourrait-il finalement qu'elle vienne camoufler la perception d'un problème qui s'aggrave et ne retarde l'adoption d'autres ures fondamentales?

\section{LA PRESCRIPTION DU COMMERCE DE L'EAU} VIRTUELLE : QUELLES IMPLICATIONS ?

Le commerce de l'eau virtuelle est, comme nous le verrons, une stratégie encore fort peu utilisée. Son potentiel d'utilisation, quant à lui, est limité par les conditions d'accès qu'il impose : le commerce de pas accessible à tous les pays. Devrait-on alors chercher des moyens pou faciliter cet accès ? Puisque cette question pourrait bien un jour se poser, elle montre toute bien un jour se poser, elle montre toute
l'importance d'analyser le potentiel réel du l'importance d'analyser le potentiel réel du commerce de l'eau virtuelle pour faire face aux éventuels effets pervers.

Le rôle de la rareté de l'eau dans les échanges internationaux

Comme il l'a été démontré précédemment, de nombreux pays en situation de pénurie d'eau tendent à augmenter leurs importations de denrées agricoles, particulièrement au MoyenOrient et en Afrique du Nord illustran Nord, illustran potentiellement une stratégie de commerce de leau vituelle pour compenser ou contrer leur tant, une observation plus attentive permet de constater que le rôle de la rareté de l'eau dans les échanges agricole internationaux demeure mineur. Si on regarde le cas de l'Amérique du Nord, par exemple, on constate que les Etats-Unis sont un exportateur net d'eau virtuelle et le Mexique un importateu (Hoekstra et Hung, 2002). Doit-on attribuer cet échange au fait que les États-Unis possèdent beaucoup d'eau et que le Mexique en a moins ? En regardant de plus près, on constate que même si les Etats-Unis possèdent beaucoup d'eau'23, un pourcentage important des recettes d'exportation céréales du partant des recettes d'exportation dans les États de l'Ogallala, sur une terre arid irriguée par une nappe phréatique surexploité (De Villiers, 2000). Ces échanges s'expliquent en fait beaucoup plus facilement par les bas prix qu'offre l'agriculture fortement subventionnée des Etats-Unis facilitée par les accords commerciaux que par l'abondance ou la rareté de l'eau.

Une étude de De Fraiture et al. (2004) vient de Uémontrer que la majorite des échanges agricoles e pron elić a la dispour des raisons qui ne sont pas relieurs relies à la disponibilite de l'eau et ont d'aileurs e commerce de l'eau virtuelle serait davantage une consequence du commerce international qu'un objectif en soi. En 1995, seulement $23 \%$ du commerce de céréales se produisaient de pays abondants en eau vers des pays en pénurie. Quan a la proportion d'eau destinée à produire des céréales pour un commerce relié aux pénuries d'eau, elle ne serait que de $2 \%$. De nombreux importateurs majeurs, tels le Japon et la Corée, ne vivent pas de pénurie d'eau (De Fraiture et al, vivent pas de penurie d'eau (De Fraiture et al. 2004). Le Japon doit importer de la nourriture non pas en raison d'une rareté de l'eau, mais plutôt d'une rareté de ses terres agricoles (Oki et al., 2003). Parmi les facteurs pouvant expliquer les importations, on note aussi les contraintes au niveau de la main-d'œuvre et les raisons politiques (De Fraiture et al., 2004).

Dans les rares cas où le commerce agricole est effectué pour des raisons reliées à la rareté de l'eau, il concerne des pays où la pénurie est extrêmement sévère. Ces pays ont alors peu d'autres choix que d'importer. Ainsi, on ne peut pas dire qu'ils économisent de l'e qu'ils economisent de l'eau en important de la nourriture : ils ne disposaient deja pas de ces ressources si bien que diminuer leurs importation de denrees se traduirait pas la famine ou une diminution de la population (De Fraiture et al. 2004).

Si on entend par commerce de l'eau virtuelle une stratégie consciente dans laquelle la disponibilité de l'eau est un facteur déterminant des échanges commerciaux, on peut donc considérer que cette stratégie est peu appliquée dans les faits. On peut alors se demander s'il s'agit d'une stratégie envisageable comme politique de redistribution mondiale de l'eau.

\section{Le commerce de l'eau virtuelle : une stratégie} accessible à tous les pays en pénurie ?

Le commerce de l'eau virtuelle pourrait-il être utilisé comme politique de gestion de l'eau à 
l'échelle mondiale ? II faudrait d'abord pour cela que les différents pays du monde y aient accès. Notre analyse montre plutôt que si cette stratégie est effectivement possible, elle n'est malheureusement pas accessible à tous en raison malheureusement pas accessib
des conditions qu'elle impose.

La possibilité, pour un pays en pénurie ou en voie de l'etre, de se procurer des denrees alimentaires par importation plutôt que de les produire luimême n'est pas accessible à tous, elle depend en fait d'un ensemble de facteurs. Tout d'abord, des surplus doivent être accessibles sur les marchés mondiaux. Si, présentement, de tels surplus existent, rien ne garantit que la situation restera même dans l'avenir. En raison de nombreux problem de qualité (pollution, salinisation problèces de qualite (pollution, salinisation) jumelés à un accroissement de la demand tation du niveau de vie), certains chercheurs croient que l'eau douce de la planète ne suffira éventuellemen plus pour répondre aux besoins alimentaire mondiaux (Postel, 1996 ; Brown, 1996). Si tous $\mathrm{n}$ sont pas si pessimistes, on peut néanmoin affirmer que les connaissances actuelles ne permettent pas d'éliminer complètement cette hypothèse.

En supposant l'existence de surplus alimentaire sur les marchés mondiaux l'accès à ceux-ci n'est possible que pour les pays qui disposent des moyens financiers nécessaires pour se les procurer. Certains auteurs estiment que le transfe de l'eau du secteur agricole vers d'autres secteurs de l'économie s'avère une politique rentable. Pour un volume donné, l'eau ainsi transféree peut produire une valeur économique 70 fois plus grande (Ohlsson, 1999, in Turton, 2000). Les importants surplus générés par le transfert d'eau du secteur agricole à d'autres secteurs de l'économie peuvent ensuite être utilisés pour importer l'eau virtuelle nécessaire pour le pays nouveaux secteu nouveaux sect.urs d'exportan qui genereront des revenusetfourniront des emplois plus tard, un pays a besoin de devises maintenant (Jaeger 2001). Pour cette raison, ce sont principalement les pays ayant déjà une économie forte et diversifiée qui adoptent, ou sont en mesure d'adopter, un politique axée sur l'importation d'eau virtuelle.

L'étude réalisée par Turton (1998) concernant le bassin du Zambeze illustre bien ce phénomène. Dans cette étude Turton cherchait à comprendre comment les différents pays du bassin du Zambèze pourraient coopérer entre eux dans une stratégie de commerce d'eau virtuelle. Les résultats montrent que cette coopération ne serait bénéfique que pour certains de ces pays : I'Afrique du Sud, le Zimbabwe, le Botswana, I'Angola, I Zambie et la Namibie ${ }^{24}$. Quant au Malawi, au Mozambique et à la Tanzanie, ils ne seraient pas même de profiter du commerce de l'eau virtuelle parce qu'ils ont une économie faible combinée à une efficacité agricole et industrielle déficiente. Or, ces trois pays subissent un risque de pénurie lorsqu'on considère le rapport entre leur tendance démographique et leurs ressources en eau (Turton, 1998). Non seulement cette étude montre l'impossibilité pour certains pays de recourir à une stratégie de commerce de l'eau virtuelle, mais elle fait ressortir un problème qui peut toucher des régions entières, comme c'est le cas pour le bassin du Zambèze. D'abord, la réussite de la stratégie est conditionnée par la mise en place de mesures permettant de protéger les échanges agricoles à l'intérieur de la région contre l'entrée sur ce marché local de denrées meilleur marché

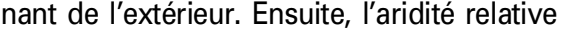
du bassin du Zambèze fait que la coopération ne saurait suffire à répondre aux besoins des différents pays. Or, même les économies les plus fortes du bassin sont peu compétitives sur le marché global et n'auraient donc pas la capacité économique de se procurer les denrées necessaires pour compenser le déficit de la région (Turton, 1998).

De plus, la dépendance vis-à-vis du commerce agricole mondial augmente l'exposition et la sensibilité aux fluctuations du marché (Warner, sensibilite aux flyctions 2003). Si les prix venaient à augmenter, même les pays possedant presentement des ressources financières suffisantes pour augmenter la part des mportations alimentaires dans leur économie pourraient voir leur situation changer. Plusieurs facteurs sont susceptibles d'influencer le prix des denrées agricoles sur les marchés mondiaux, affectant la capacité de nombre de pays à se les procurer : on compte parmi ceux-ci la libéralisation des échanges et la hausse de la demande mondiale.

Actuellement, l'importation de produits agricoles est grandement facilitée par le prix de ceux-ci. En 1997, en raison des très fortes subventions à l'agriculture en Europe et en Amérique du Nord, le prix des céréales était de 140 \$ la tonne alors qu'il en coûtait 200 pour la produire (Allan, 1996). en coûtait 200 pour la produire (Allan, 1996). D'après le modèle de Ramirez-Vallejo et Rogers
(2004), dans un contexte de complète (2004), dans un contexte de complète libéralisation du commerce agricole, avec retrait des subventions à l'agriculture et des barrières tarifaires, le prix du riz augmente
2020 , et le prix du bœuf de $18 \%$.

Une hausse de la demande agricole mondiale aurait aussi pour effet de faire augmenter les prix Cette hausse par 'augme have par une L'entrée de la Chine et de l'Asie marché global des céréales pourrait affecter de façon importante les pays importateurs tels que les pays du Moyen-Orient (Allan, 1996a). Ces régions, qui comptent $40 \%$ de la population de Jordanie ont tout deux craint qu'une guerre n'éclate au Moyen-Orient en raison son déficit en eau (De Villiers, 2000). Pourtant, ces guerres de l'eau n'ont pas eu lieu, qui pourrait s'expliquer par commerce de l'eau viquer par

19 - C'est à dire un concept qui prescrit, qui prétend qu'on "devrait faire ceci pour cela".

20 - Parmi ceux-ci, on compte le Conseil Mondial de l'eau, la FAO (Organisation des Nations Unies pour l'alimentation et Unies pour I'alimentation e l'agriculture), I'UNESCO-IH (Institute for Water Education et I'IWMI (International Wat
Management Institute).

21 - Ainsi, la production d'un kilogramme de maïs en Égypt nécessite $1,12 \mathrm{~m}^{3}$ d'eau, alors que la même quantité produite en France n'en demande que $0,6 \mathrm{~m}^{3}$ (Renault, 2003).

22 - Pour une même quantité d'eau, il est alors possible d'obtenir un profit plus grand.

23 - Ils disposent de $9000 \mathrm{~m}^{3}$ d'eau par habitant par année (De Villiers, 2000)

24 - Certains de ces pays bénéficieraient d'une importation d'eau virtuelle, d'autres d'une exportation. 
25 - L'Inde serait le $5^{e}$ plus important pays exportateur net d'eau virtuelle, avec des exportations de $161,1 \mathrm{~km}^{3}$ d'eau par année entre 1995 et 1999 (Hoekstra et Hung, 2002).

26 - Le Bangladesh, par exemple, importe de l'eau virtuelle de l'Inde Or, ces deux pays ont un certain nomb pays ont un certain s bilatéraux olus (Parveen et Faisal, 2004). Leurs rapports de commerce étant fortement déséquilibrés, une réponse du Bangladesh en cas d'exportation discriminatoire, de politique tarifaire ou d'embargo n'aurait aucun effet sur I'Inde (Parveen et Faisal, 2004). Le Bangladesh devrait alors se tourner vers une source alternative d'approvisionnement telle la Thailande ferait face à des prix beaucoup ferait face à des prix beaucoup plus élever 2004).

27 - Ces chiffres sont tirés de De Villiers, 2000 mondiale, ont réussi à répondre aux besoins croissants de leur population depuis les années 1940 (Allan 1996a). Cette situation pourrait être appelée à changer. Déjà, entre 1995 et 1999, la Chine était le $5^{\mathrm{e}}$ importateur mondial net $\mathrm{d}^{\prime}$ eau virtuelle, avec une importation de $101,9 \mathrm{~km}^{3}$ par année (Hoekstra et Hung, 2002). La croissance de sa population, la hausse de son niveau de vie et les fuites dans le réseau contribuent à une demande croissante (Lasserre, 2005). L'Inde, actuellement exportatrice ${ }^{25}$, pourrait aussi voir sa situation s'inverser. Tandis que le barrage est le principal moyen d'approvisionnement de ce pays, principal sinverser. Tandis que le barrage est la construction de nouveaux barrages est surveillee de press par des groupes locaux et internationaux (Allan, 1996a). Bien que de moussons favorables lui aient permis d'accumule des stocks dans les dernières années, il pourrait lui être difficile d'atteindre les 200 millions de tonnes qui lui seront nécessaires annuellement dans la décennie à venir (Allan, 1996a).

Enfin, le système commercial international est dominé par de puissants intérêts qui peuvent être difficiles à contrôler pour les pays les plus pauvres (Warner, 2003). Les multinationales alimentair peuvent manipuler les prix en gar peuvent manipuler les prix en gardant de gigantesques stocks (Warner, 2003). Or, ce multinationales détiennent déjà une large part du marché. Au niveau du commerce international du grain, par exemple, deux compagnies multinationales contrôlent de 70 à $80 \%$ du marché (Norberg-Hodge et al., 2002). La manipulation des prix pourrait réduire l'accès de nombreux pays au commerce de l'eau virtuelle.

En plus de facteurs économiques, des facteurs politiques pourraient aussi menacer la capacité d'un pays de poursuivre ou d'augmenter ses d'un pays de pourd 're d'augmenter ses importations. D'abord, l'importation centralisée de grain bon marché pourrait créer un monopole sur le marché alimentaire (Warner, 2003). La nourriture pourrait ensuite être distribuée aux villes selon leur allégeance politique. Dans le même sens, la sécurité alimentaire des États dépendrait de leur allégeance à la politique extérieure américaine, créant une dépendance politique que certains pourraient qualifier de néocoloniale (Warner, 2003). De plus, pour les pays en situation de dépendance, la sécurité alimentaire pourrait se voir menacée par l'utilisation d'un embargo alimentaire comme arme politique ${ }^{26}$.

Vers une meilleure réponse aux besoins de base des populations et des écosystèmes ?

Nous avons pu voir que la stratégie de commerce de l'eau virtuelle n'est possible que pour certain pays, et que son accessibilité pourrait se réduire encore davantage à l'avenir. Toutefois, puisque pour certains pays il s'agit d'une stratégie accessible et que d'autres pourraient se la voir imposer, il est fondamental d'évaluer le potentiel réel du commerce de l'eau virtuelle pour répondre aux défis posés par la rareté de l'eau.

En supposant qu'un certain nombre de pays en situation de pénurie ou en voie de l'être soient capables d'augmenter la part d'importations agricoles dans leur économie, peut-on s'attendre à ce que le commerce de l'eau virtuelle favorise une meilleure réponse aux besoins de base de populations et des écosystèmes ? II va de soi que pour des pays ne disposant pas des $1100 \mathrm{~m}^{3}$ par personne nécessaires pour cultiver le minimum de nourriture requise pour dépasser le seuil de la malnutrition 27 , le recours aux importation alimentaires est une condition indispensable à répone aux besoins de base de leurs habitants. Par contre il nesoins habitants. Par contre, il ne s'agit pas de la seule. Un pays pourrait tres bien importer des quantité importantes de produits alimentaires sans que ceux-ci soient distribués équitablement dans sa population. Plusieurs pays qui disposent d'ailleurs de suffisamment d'eau pour nourrir leur population rencontrent tout de même des problèmes de sous-alimentation. En Thaillande, le $3^{\text {e }}$ plus important exportateur net d'eau virtuelle dans le monde (Hoekstra et Hung, 2002), l'eau n'est pas rare. Pourtant, pour la période allant de 1999 et $2001,19 \%$ de la population y était sousalimentée (Organisation des Nations Unies pour alimentee (Organisation des Nations Unies pou l'alimentation et l'agriculture, 2003). Le même pourcentage de sous-alimentation prevaut au Viêt$\mathrm{Nam}, 7^{\mathrm{T}}$ exportateur mondial d'eau virtuelle. Au Guatemala, $9 \mathrm{e}$ exportateur mondial d'eau virtuell (Hoekstra et Hung, 2002), c'est $25 \%$ de la population qui est considérée comme sousalimentée.

Dans le cas des pays qui sont encore capables de subvenir à leurs besoins agricoles, l'importation d'eau virtuelle peut se présenter comme un moyen de diminuer les risques de pénurie et de libérer de l'eau pour les besoins de la population et libérer de l'eau pour les besoins de la population et
des écosystemes. Toutefois, rien ne garantit que la nourriture importée et l'eau libérée par ces importations seront destinées prioritairement aux besoins de base de la population et des écosystèmes. Dans le bassin de I'Usangu, par exemple, les cultures irriguées fournissent des revenus à 30000 familles, pour un montant tota de 15.9 millions SUS (Kadigi et al 2004). II peut être difficilo pour (Kadigi et al., 2004). II peut etre difficile pour ces paysans de trouver un emploi leur permettant de se nourri convenablement, d'autant plus que les bas salaire constituent "lavantage comparatif" des pays du tiers-monde (Rosset, 2002). Quant à l'eau que les importations libèrent, à quels usages sera-t-elle destinée ? A la population et aux écosystèmes ? Pour financer ses importations de produit agricoles, un pays doit trouver de l'argent. Pour ce faire, il devra utiliser l'eau libérée à des fins industrielles, dans l'industrie touristique par exemple. En Indonésie, on a pu constater un exemple parlant d'une eau dirigée vers les usages 
les plus rentables plutôt que vers les besoins les plus fondamentaux. Alors qu'en 1994, I'Indonésie souffrait d'une grave sécheresse, les terrains de golf de Jakarta ont continué à recevoir $1000 \mathrm{~m}^{3}$ d'eau par terrain pendant que les puits de ésidents étaient à sec (Barlow et Clarke, 2002). L transformation de terres agricoles en terrains de golf pourraient fort bien résulter d'une politique de 'eau qui intègre le commerce de l'eau virtuelle, car elle consiste effectivement à utiliser l'eau pour les usages les plus rentables.

'eau libérée peut aussi servir à produire de denrées agricoles à haute valeur ajoutée destinées à l'exportation. C'est le cas aux Philippines, un pays qui oscille entre les importations et les pays qui oscille entre les importations et les exportations d'eau virtuelle (Hoekstra et Hung 2002) et dont la part de production locale est principalement destinée à l'exportation. Dans ce pays, les subventions gouvernementales ne vont as à la riziculture, mais à la production d'orchidées, d'eucalyptus, de mangues et d'asperges. En parallèle, environ 28 millions de Philippins n'arrivent pas à assurer leurs besoins alimentaires de base (Cainglet, 2002). Quant aux cosystèmes, quelle part leur reviendrattil? protection des ecosystemes etant rarement un des re pourt se trouver bien lon dans la liste des pile pourrait se trouver bien loin dans la liste des priorités d'un pays qui doit générer des revenus pour financer ses importations.

a protection des écosystemes des pays disposant de beaucoup d'eau est quant à elle évacuée du discours sur le commerce de l'eau virtuelle. Celuici est basé sur le postulat que certains pays sont en pénurie d'eau tandis que d'autres pays affichen des surplus. Dans cette perspective, toute l'eau qui s'écoule vers la mer est perdue. II s'agit d'une sonception de l'eau qui néglige de consid́c d'une conception de l'e qui perdue. Il s'agit d'une efet, l'eau n'a pas pour seule fonction de répondr ux besoins domestiques, industriels et agricoles des êtres humains. L'eau transporte aussi de substances nutritives vers la mer, elle soutient les pêcheries, protége les zones humides, filtre les polluants, sert d'habitat à une riche biodiversité et maintient un équilibre au niveau des sels et des sédiments (Postel, 1996).

Les pays qui, dans une logique de commerce de l'eau virtuelle, devraient jouer le rôle d'exportateurs, peuvent effectivement posséder présen(liven ement plus deau quils $n$ 'en ont besoin pour leurs usages humains. Cependant, leurs ecosystemes par exemple, le Gange ne se jette plus à la mer pendant certaines parties de l'année. Le manque d'eau douce qui coule vers la mer cause une avancée de l'eau salée dommageable pour I'habitat des palétuviers et des poissons du delta ${ }^{30}$ (Postel, 1996). Aux États-Unis, premier pay exportateur d'eau virtuelle (Hoekstra et Hung,
2002) I'État de la Californie a perdu 95\% de ses terres humides et les populations d'oiseaux migratoires et de sauvagines qui en dépendaient migratoires et de sauvagines qui en dépendaient ont diminué de 60 millions
aujourd'hui (Postel, 1996).

L'eau n'étant pas le seul facteur de production des biens importes, les consequences du commerce de l'eau virtuelle sur les ecosystemes des pays exportateurs dépassent donc la seule question de l'eau. Ainsi, le Brésil, qui dispose de beaucoup d'eau, semble offrir un potentiel intéressant pour exporter de l'eau expres alimentares. Toutefois, le sol aussi constitue un facteur de production de l'agriculture, et une augmentation de la production au Brésil demanderait d'utiliser de nouvelles terres, ce qui pourrait se faire au détriment de la conservation de la forêt amazonienne. En restant toujours dans l'exemple de l'agriculture, les engrais chimiques, la machinerie et les semences génétiquement modifiées constituent aussi des facteurs de production pouvant avoir des conséquences sur l'eau et sur les écosystèmes. Les principaux pays exportateurs de denrées agricoles ne sont pas nécesarement ceux qui ont les pratiques los plus 作 par par exemple, parmi les cinq principaux pays exportates plus importants producteurs de cultures génétiquement modifiées, soient respectivement les Etats-Unis, I'Argentine et le Canada $^{32}$ (Genewatch, 2002). On ne parle pourtant pas de sols virtuels, d'engrais chimiques virtuels et d'organismes génétiquements modifiés virtuels échangés par le commerce international ${ }^{33}$.

Ainsi, il semble peu probable qu'une politique de l'eau intégrant le commerce de l'eau virtuelle favorise un usage de l'eau axé sur la reponse aux besoins de base des gens et de l'envirc cur aue cu exportateurs. Cette strategle, en recourant au commerce international, se prete aussi règles. Si ces règles ne favorisent pas ceux qui en auraient le plus besoin, et qu'on remarque parfois e contraire, on pourrait s'attendre à ce qu'il en soit de même du commerce de l'eau virtuelle.

\section{Commerce de l'eau virtuelle et perception du} risque

Le potentiel du commerce de l'eau virtuelle face aux défis posés par la gestion le l'eau serait face aux donc (effets pervers? Ce pourrait être le cas s'il empêchait indirectement que ne soient prises d'autres mesures qui s'imposent pour faire face aux pénuries et pour les limiter.

De nombreux moyens existent pour réduire la controle des naissances et la modification du regime alimentaire pourraient permettent de diminuer la pression sur la
28 - Les Philippines étaient exportateurs nets entre 1995 et 1996 et importateurs entre 1997 et 1999.

29 - L'Inde est considérée comme le 5 e plus important exportateur d'eau virtuelle (Hoekstra et Hung, 2002).

30 -Ces espèces constituent d'ailleurs une ressource alimentaire importante pour les habitants locaux (Postel, 1996).

31 - Ces cinq pays sont les Etats-Unis, le Canont Thailande l'Argentine et I' Thailande, I'Argentin

32 - Ces trois pays étaient les plus importants producteurs de cultures génétiquement modifiées en 2001.

33 - Merett (2003) a d'ailleurs remarqué que le terme virtue est trompeur. Pour lui, concept n'arrive pas à bie représenter la réalité puisqu' sous-entend que de l'eau est transféree entre deux est transferee entre deux pays, alors qu'en realé, ce sont des transferts de nourriture qui on lieu. L'expression évacue alor un ensemble d'autres dimensions de l'agriculture qui, san être reliées à l'eau, sont intimement reliées aux produits agricoles échangés les caractéristiques du sol, les droits de propriété et le budge des fermes (Merett, 2003). 
34 - II s'agit d'une technique d'irrigation basée sur des faibles flux d'eau.

35 - Le taux de rendement de l'aspersion goutte-à-goutte se situe entre 70 et $90 \%$ (Lasserre, 2002). ressource. Une politique visant à réduire la demande pourrait aussi inclure des incitations et des technologies pour réduire les usages domestiques (tels que des incitations à l'achat de toilettes qui consomment moins d'eau), la rénovation des aqueducs municipaux qui fuient $e$ le recyclage des eaux usées (Lasserre, 2002a). Différents moyens permettent aussi de réduire la demande en eau du secteur agricole, qui est à l'origine d'environ 70 à $75 \%$ des prélèvements (Lasserre, 2002a). L'efficacité hydraulique peut d'abord être accrue par l'irrigation au goutte-àgoutte ${ }^{34}$. Alors que dans l'irrigation par gravité, la technique d'irrigation la plus répandue, $60 \%$ de l'eau est perdue, les pertes 35 avec le goutte-àgoutte se situent entre 10 et $30 \%$. Nomb d'autres goutte se situent entre 10 et $30 \%$. Nombre d'autres mesures permettent de diminuer les pertes en eau dans le secteur agricole : la production de culture sous couvert ombragé et l'utilisation de paillis (Wallace, 2000) n'en sont que quelques exemples. Dans la majeure partie des pays importateurs, il y a encore une latitude suffisante pour améliorer la productivité de l'eau utilisée, c'est-à-dire pour produire davantage avec chaque goutte d'eau ( $D e$ Fraiture et al., 2004).

La mise en place de telles mesures de réduction de a demande nécessite cependant une certain volonté politique. volonte politique. Le coût des réformes des politiques de l'eau, telles que la reglementation, est élevé (Allan, 2000). Politiquement silencieuse (Allan, 1996), la stratégie de commerce de l'eau virtuelle pourrait permettre aux décideurs d'affronter une situation de pénurie ou de surutilisation de la ressource sans avoir à mettre en place des politiques financièrement ou politiquement coûteuses. On peut alors se demander si, globalement, le commerce de l'eau virtuelle ne contribue pas à retarder la mise en place de politiques favorisant une utilisation de I'eau plus durable. D'ailleurs, dans la région du Moyen-Orient et d'Afrique du Nord, les décideurs se sont déà pris au piege du discours sécurisant. se sont deja pris au piege du discours securisant La perception du risque est manipulée par ce discours, et la difference entre le risque et sa perception s'agrandit. Le risque continue à exister mais sans que ne soient déployés d mécanismes pour y faire face (Allan, 2000).

Finalement, le commerce de l'eau virtuelle comme stratégie de gestion de l'eau est non seulement peu appliqué, mais aussi peu applicable. Nombre de pays n'y ont pas accès et cet accès pourrait être réduit encore davantage à l'avenir pour des raisons politiques et économiques. Le potentiel du commerce de l'eau virtuelle face aux défis posés commerce d'é l'eau mince lorsqu' on considère les modes actuels de distribution de I'eau et de production des denrées de base. I serait même possible que plutôt que de régler le problème, le commerce de l'eau virtuelle ne fasse qu'assurer la reproduction de modes de production et de consommation qui menacent la pérennité de la ressource. Le jeu des échanges, en donnant la fausse impression que la situation s'améliore, pourrait alors retarder la recherche de solutions politiquement plus difficiles à prendre, mais pourtant indispensables dans un contexte de pénurie croissante.

\section{Conclusion}

Le concept d'eau virtuelle n'est pas sans intérêt bien au contraire. En rendant compte de l'apport et du contenu en eau de mettre liffér differents modes de consommation. Il permet assi de tenir compte des transferts d'eau induits par le commerce international. Cette considération permet à son tour de réaliser toute l'importance que jouent les politiques commerciales compte tenu des pénuries d'eau que vivent plusieurs pays. Le commerce de l'eau virtuelle pourrait enfin permettre aux analystes, politiciens et aux citoyens de prendre conscience que les importations et exportations de denrées intensives en eau ne sont pas sans conséquences sur le plan écologique social et environnemental.

Face à l'inégale répartition des ressources et à la menace croissante de pénurie, avec toutes les conséquences que cela implique, il peut être tentant de proposer le commerce de l'eau virtuell comme politique efficace de redistribution. Toutefois, devant un problème aussi complex que l'approvisionnement, la disponibilité et la distribution de l'eau, les solutions le sont aussi. Le concept d'eau virtuelle permet de mettre en lumière le potentiel du commerce internationa dans la répartition des ressources en eau : il en montre aussi les limites. Le marché eau , il en montre aussi les limites. Le marché est accessible ceux qui disposent des ressources financières penurie ne les possed qui n'en possedent pas suffisamment pour subir es hausses de prix que pourrait provoquer une augmentation de la demande ou la libéralisation du commerce.

$\mathrm{Si}$, sur l'ensemble de la planète, l'eau était destinée en priorite aux besoins de base de la population en second lieu à ceux des écosystèmes, le commerce de l'eau virtuelle pourrait offrir de nouvelles possibilités pour rendre possible une distribution équitable malgré les différence une istribution equitable malgre les differences entre es ressources de chaque pays. Toutefois, si on egarde les modes de distribution actuels, ils semblent plutôt refléter les rapports économiques t rien ne laisse croire qu'il en serait autremen avec le commerce de l'eau virtuelle. Pour certain pays, la question ne se pose pas : ils ne possèden déjà plus l'eau nécessaire pour survivre et doiven nécessairement importer des biens intensifs en eau, donc faire du commerce de l'eau virtuelle. Pour les autres, les promesses du commerce de eau virtuelle demandent à être scrutées avec la 
plus grande attention : elles pourraient bien s'avérer fort trompeuses.

Le concept d'eau virtuelle peut donc être considéré comme un concept à la fois utile et dangereux. II peut être utile pour mieux comprendre comment le commerce international affecte les ressources en eau. II devient toutefois dangereux dès qu'il tend à être normatif, car les limites qu'il port peuvent alors se répercuter dans des décision politiques. La dimension normative du concep pourrait d'ailleurs être appelée à s'intensifier si la prescription d'une stratégie de commerce de l'eau virtuelle bénéficiait aux pouvoirs dominants. pourrait s'avérer le cas puisque le concept perme d'évacuer des questions de fond sur lorigine de l'épuisement des ressoures en des inéglíne de l'epuisem

\section{BIBLIOGRAPHIE}

ALLAN J.A., 1996, Virtual water : a strategic resource : global solutions to regional deficits, Ground Water, Vol. 36, n 4, p. 545-546.

ALLAN J.A., 1996a, The political economy of water : reasons for optimism but long term caution, in ALLAN J. A. (dir.), Water, Peace and the Basin, Londres, I.B. Tauris, p. 75-119.

ALLAN J.A., 1997, 'Virtual water' : a long term solution for the water short Middle Eastern economies ? Occasional paper 3, School of oriental and African studies (SOAS), University of London, 20 p., http://web.macam.ac.il/ arnon/IntME/water/OCC03.PDF

ALLAN J.A., 2000, Contending environmenta knowledge on water in the Middle East : global regional and national contexte, in STOTT $P$. et SULLIVAN S. (eds), Political Ecology : Science, Myth and Power, New York, Oxford University Press, p. 117-131.

ALLAN J.A., 2001, The Middle East Water Question : Hydropolitics and the Global Economy, Londres, I.B. Tauris, 382 p.

ALLAN J.A., 2003, Virtual Water - the water, food trade nexus : useful concept or misleading metaphor? Water International, Vol. 28, n 1 , p. 106-113.

BARLOW M. et T. CLARKE, 2002, L'or bleu : I'eau nouvel enjeu stratégique et commercial, Montréa Boréal, $390 \mathrm{p}$.

BOUWER H, 2000, Integrated water management : emerging issues and challenges, Agricultura Water Management, Vol. 45, p. 217-228.

BROWN L, 1996, Tough choices: facing the challenge of food scarcity, Washington $D C$, World Watch Institute, Environmental alert series, $159 \mathrm{p}$.
CAINGLET J, 2002, Orchidées, fruits exotiques et ventres creux, L'écologiste, Vol. $3, n^{\circ} 7$, p. 67-69.

CHAPAGAIN A.K. et A.Y. HOEKSTRA, 2003, Virtual water trade : a quantification of virtual water flows between nations in relation to international trade of livestock and livestock products, in HOEKSTRA A.Y. (dir.), Virtual Water Trade : Proceedings of the International Expert Meeting on Virtual Water Trade, Value of Water Research Report Series $\mathrm{N}^{\circ} 12$, Delft (the Netherlands), UNESCO-IHE, p. 49-

CHAPAGAIN A.K et HOEKSTRA A.Y, 2004, Water Footprints of Nations, volume 1: Main Report, Footprints of Nations, volume 1 : Main Report, Value of Water Research Report Series n ${ }^{\circ} 16$, Delf (the Netherlands), UNESCO-IHE Institute for water education, $76 \mathrm{p}$.

De FRAITURE C., CAI X., AMARASINGHE U., ROSEGRANT M. et MOLDEN D., 2004, Does international cereal trade save water ? The impact of virtual water trade on global use, Comprehensive assessment, Research report $n^{\circ} 4$, Colombo, Sri Lanka, Comprehensive Assesment Secretariat, $27 \mathrm{p}$.

DE VILLIERS M., 2000, L'eau, Montréal, Leméac, $437 p$

FALKENMARK M., 1997, Meeting water requirements of an expanding world population. Philosophical Transactions of the Royal Society of Philosophical Transactions of the Royal Society of
London Series B-Biological Sciences, Vol. 352, p. $929-936$.

GENEWATCH, 2002, Genetic engineering : review of developments in 2001, Briefing $\mathrm{n}^{\circ} 17$. http://www.genewatch.org/publications/Briefs/brie f17.pdf

HOEKSTRA A.Y., 2003, Virtual water : An introduction, in HOEKSTRA A.Y. (dir.), Virtual Water Trade : Proceedings of the International Expert Meeting on Virtual Water Trade Value of water research report series $\mathrm{N}^{0} 12$, Delft (the Netherlands), UNESCO-IHE, p. 13-23.

HOEKSTRA A.Y. et HUNG P.Q., 2002, Virtual water trade : A quantification of virtual water flows between nations in relation to international crop trade Value of Water Research Report Series $N^{\circ} 11$, Delf (the Netherlands), UNESCO-IHE Institute for water education, $66 \mathrm{p}$.

HOEKSTRA A.Y. et HUNG P.O., 2005 , Globalisation of water resources : international virtual water flows in relation to crop trade, Global Environmental Change, Vol. 15, $\mathrm{n}^{\circ} 1$, p. 45-56.

JAEGER C.C., 2001, Challenge of global water management, in EHLERS E. et KRAFFT T. (éds), Understanding the Earth System Compartments, Processes and Interactions, Springer, p. 125-135. 
KADIGI R., JAPHET M.J., KASHAIGILI J. et MDOE N.S., 2004, The economics of irrigated paddy in Usangu Basin in Tanzania : water utilization, productivity, income and livelhood implications. Physics and Chemistry of the Earth, Vol. 29, p. $1091-1100$.

LASSERRE F., 2002, Les guerres de l'eau : mythe ou réalité future? in LASSERRE $F$. et DESCROIX L. (dir.), Eaux et territoires: Tensions, coopérations et géopolitique de l'eau, Sainte-Foy (Québec), Presses de l'Université du Québec, p.17-72.

LASSERRE F., 2002a, L'eau rare ? Des solutions pour assurer l'approvisionnement, in LASSERRE $F$. et DESCROIX L. (dir.), Eaux et territoires : Tensions, coopérations et géopolitique de l'eau, Sainte-Foy (Québec), Presses de l'Université du Québec, p. 73114.

LASSERRE F., 2005, Quand le dragon chinois a soif : le défi de l'eau en Chine, Options politiques, juillet-août, p. 8-12.

MERRETT S. 2003. Virtual water and Occam's razor, Water International, Vol.28, n 1, p. 103-105.

NORBERG-HODGE H., MERRIFIELD T. et GOERELICK S., 2002, Bringing the Food Economy Home : Local Alternatives to Global Agrobusiness, West Hartford, Kumarian Press, $150 \mathrm{p}$.

OKI T., SATO M., KAWAMURA A., MIYAKE M., KANAE S. et MUSIAKE K., 2003, Virtual water trade to Japan and in the world, in HOEKSTRA A.Y. (dir.), Virtual Water Trade: Proceedings of the International Expert Meeting on Virtual Water Trade Value of Water Research Report Series $N^{\circ} 12$, Delft (the Netherlands), UNESCO-IHE, p. 221233.

OKI T. et S. KANAE, 2004, Virtual water trade and world water resources, Water, Science and Technology, Vol. 49, n 7, p. 203-209.

ORGANISATION DES NATIONS UNIES POUR L'ALIMENTATION ET L'AGRICULTURE, 2003 L'état de l'insécurité alimentaire dans le monde 2003 : Suivi des progrès accomplis en vue de la 2003 : Suivi des progres accomplis en vue de la
réalisation des objectifs du Sommet mondial de realisation des objectifs du Sommet mondial de
l'alimentation, Rome, Organisation des Nations I'alimentation, Rome, Organisation des $\mathrm{Nat}$.
Unies pour I'alimentation et I'agriculture, $40 \mathrm{p}$.

PARVEEN S. et FAISAL I.M., 2004, Trading virtual water between India and Bangladesh : a politicoeconomic dilemma, Water Policy, Vol. 6, $n^{\circ} 6$, p. 549-558.

POSTEL S., 1996, Dividing the waters : Food security, ecosystem health and the new politics of scarcity, Washington, World Watch Papers 132 $76 \mathrm{p}$
OADIR M., BOERS T. M., SCHUBERT S GHAFOOR A. et MURTUZA G., 2003, Agricultural water management in water-starved countries : hallenges and opportunities, Agricultural Water Management Vol. 62, n³, p. 165-185.

RAMIREZ-VALLEJO J. et ROGERS P., 2004, Virtua water flows and trade liberalization, Water Science and Technology, Vol. 49, $n^{\circ}$ 7, p. 25-32.

RENAULT D., 2003, Value of virtual water in food: principles and virtues, in HOEKSTRA A.Y (dir.) Virtual Water Trade: Proceedings of the Virtual Water Trade: Proceedings of the International Expert Meeting on Virtual Wate Trade, Value of Water Research Report Series 91.

RENAULT D. et WALLENDER W.W., 2000 Nutritional water productivity and diets, Agricultural Water Management, Vol. 45, $n^{\circ} 3$, p. 275-296.

ROSSET P., 2002, Le mythe des avantages comparatifs, L'Ecologiste, Vol. 3, n 1, p. 34-35.

SECRÉTARIAT DU TROISIÈME FORUM MONDIAL DE L'EAU ET CONSEIL MONDIAL DE L'EAU, Conclusion du 3 e Forum mondial de l'eau : 100 Conclusion du $3^{e}$ Forum mondial de l'eau : 100 Communiqué de presse Communiqué de presse,
http://www.worldwatercouncil.org/download/CP_fi http://www.worldwate
nalday_23.03.03.pdf

SHUVAL H., 1999, Sustainable water development under conditions of scarcity : Israel as a case study in MARCHISIO S., TAMBURELLI G. et PECORARO L. (éds), Sustainable Development and Management of Water Resources : a Legal Framework for the Mediterranean, Rome Institute for Lo Studies on the International Community, p. 196223.

TURTON A.R., 1998, The hydropolitics of Southern Africa: The case of the Zambezi River Basin as an area of potential co-operation based on Allan's concept of 'virtual water', Mémoire de maîtrise Johannesburg, University of South Africa, 272 p.

TURTON A.R., 2000, A strategic decision-makers guide to virtual water, Papier présenté à l'atelier de travail sur l'eau virtuelle en Afrique du Sud, à Maseru,

http://www.up.ac.za/academic/libarts/polsci/awiru

WALLACE J.S., 2000, Increasing agricultural water use efficiency to meet future food production, Agriculture, ecosystems and environment, Vol. 82 $\mathrm{n}^{\circ} 1$, p. 105-119.

WARNER J., 2003, Virtual water - virtual benefits ? Scarcity, distribution, security and conflicts reconsidered, in HOEKSTRA A.Y. (dir.), Virtual 
Water Trade : Proceedings of the International Expert Meeting on Virtual Water Trade, Value of Water Research Report Series No12, Delft (the Netherlands), UNESCO-IHE, p. 125-134.

YANG H. et ZEHNDER A., 2001, China's regiona water scarcity and its implications for grain supply and trade, Environment and Planning A, vol. 33, $n^{\circ} 1$, p. 79-95.

YANG H. et ZEHNDER A., 2002, Water scarcity and food import : a case study for southern Mediterranean countries, World Development, Vol. 30, n 8 , p. 1 413-1 430.
ZIMMER D. et RENAULT D., 2003, Virtual water in food production and global trade: review of methodological issues and preliminary results, in HOEKSTRA A.Y. (dir.), Virtual Water Trade : Proceedings of the International Expert Meeting on Virtual Water Trade, Value of Water Research Report Series $N^{\circ} 12$, Delft (the Netherlands), UNESCO-IHE, p. 93-107.
Adresse des auteurs

Lysiane Roch

Assistante-chercheure à la

Chaire de responsabilité sociale

et de développement durable

École des sciences de la gestion

Université du Québec à Montréa Case postale 8888, succursale Case postal
Centre-Ville

Montréal (Québec) Canada H3C

Montre

Téléphone : 514.987 .3000 \#4183 Télécopieur : 514.987.3372

E.mail :

roch.lysiane@courrier.uqam.ca Site web : www.crsdd.uqam.ca

Corinne Gendron

Titulaire de la Chaire de

responsabilité sociale et

développement durable

Professeure au département

d'organisation et ressources

humaines

École des sciences de la gestion Université du Québec à Montréa Case postale 8888, succursale Centre-Ville

Montréal (Québec) Canada H3C 3P8

Téléphone : 514.987 .3000 \#1400 Télécopieur : 514.987.3372

E.mail :

gendron.corinne@uqam.ca

Site web : www.crsdd.uqam.ca 